Research Article

\title{
The Intelligent Healthcare Data Management System Using Nanosensors
}

\author{
Ulzii-Orshikh Dorj, ${ }^{1}$ Malrey Lee, ${ }^{1}$ Jae-young Choi, ${ }^{2}$ Young-Keun Lee, ${ }^{3}$ and Gisung Jeong ${ }^{4}$ \\ ${ }^{1}$ Center for Advanced Image and Information Technology, School of Electronics \& Information Engineering, \\ Chonbuk National University, 664-14, 1Ga, DeokJin-Dong, Jeonju City, Chonbuk 561-756, Republic of Korea \\ ${ }^{2}$ Department of Computer Engineering, Sungkyunkwan University, Seoul City, Republic of Korea \\ ${ }^{3}$ Department of Orthopedic Surgery, Research Institute of Clinical Medicine of Chonbuk National University, \\ Biomedical Research Institute of Chonbuk National University Hospital 20, Geonji-ro, Jeonju 54907, Republic of Korea \\ ${ }^{4}$ Department of Fire Service Administration, Wonkwang University, Iksan City, Republic of Korea
}

Correspondence should be addressed to Malrey Lee; mrlee@chonbuk.ac.kr, Jae-young Choi; jaeychoi@skku.edu, Young-Keun Lee; trueyklee@naver.com, and Gisung Jeong; jgskor@wonkwang.ac.kr

Received 27 July 2017; Accepted 15 October 2017; Published 26 December 2017

Academic Editor: Mucheol Kim

Copyright ( 2017 Ulzii-Orshikh Dorj et al. This is an open access article distributed under the Creative Commons Attribution License, which permits unrestricted use, distribution, and reproduction in any medium, provided the original work is properly cited.

We developed a design of Intelligent Healthcare Data Management System using nanosensors (IHDMS) and composed an application for mobile device. The proposed IHDMS will coordinate the healthcare data of the patients from nanosensors and transforms it into a worldwide consumed standard HL7 (Health Level Seven) for conversion of healthcare data. This converted data dispatches to a server of its system. The battery lifetime of the facility is feasible to increase, the memory usage is less than $100 \mathrm{~KB}$, and it operates all data by employing few and far between resources. Moreover, the proposed system decreases the waiting time in the transposing data, and secured channel was used for the server of the healthcare center in the running HL7 format data.

\section{Introduction}

As with acceleration in developing technologies, intelligence healthcare systems are also moving towards novel approaches and models of healthcare based on nanosensor, smart phone, smart watch, and so on. Currently, nanotechnology is getting one of the incredible studies and plays an important role in many fields, such as electronics, telecommunication, agriculture and food, materials, energy storage, biotechnology, medicine, and healthcare system.

Intelligent Healthcare Data Management System allows a sick person or patient to use a healthcare service such as a diagnosis service, emergency management service, and monitoring service whenever and anyplace. The Intelligent Healthcare Data Management System (IHDMS) with nanosensors contains three components. Schematic of the Healthcare Data Management System using nanosensors is illustrated in Figure 1.
Component 1 involves wearable nanosensors worn by patient. Each nanosensor can recognize, represent, and process one or more physiological signals, namely, heart rate monitoring by an electrocardiogram (ECG) nanosensor, brain electrical activity observation by an electroencephalogram (EEG) nanosensor, muscle activity monitoring by an electromyogram (EMG) nanosensor, blood pressure recording by a blood pressure nanosensor, trunk position monitoring by a tilt nanosensor, and so on.

Component 2 involves an individual application, which is executing on a smart phone. This application is answerable for a considerable commitment. It greatly performs as an interface to the wireless medical sensors, which assembles the sick person or patient's health information and transfers it to a medical healthcare server. The WBAN (wireless body area network) contains network configuration and management. 


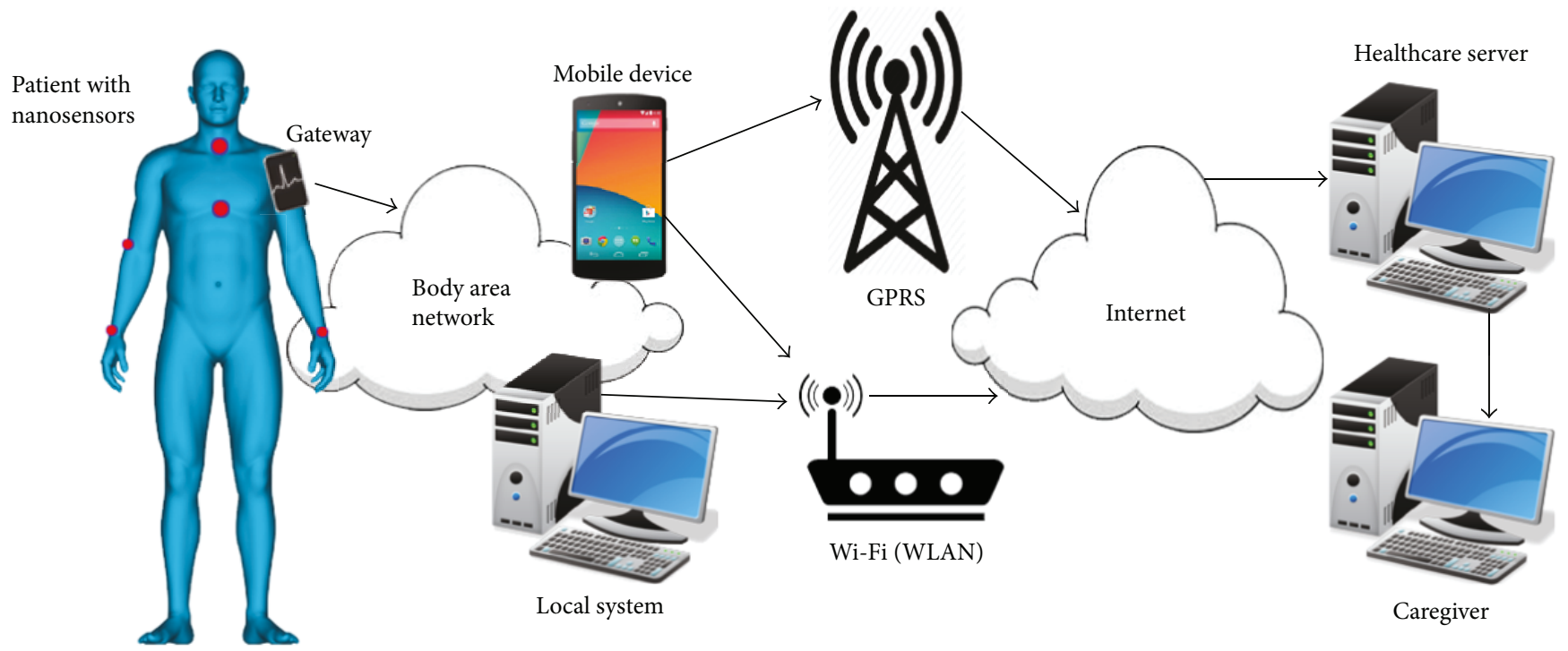

Figure 1: Schematic of the Intelligent Healthcare Data Management System using nanosensors.

The configured WBAN network is conducted by an application. It services to share channels, synchronize time, and also retrieve, process, and transfer of data. Based on the information from multiple medical sensors, the application should determine the patient's health state and provide feedback through a user friendly and intuitive graphical. Consequently, if a communication channel to the medical server is available, the application sets out a secure link to the medical server and sends reports that can be united into the patient's medical record in HL7 format. However, if a link between the mobile device and the medical server is not available, the application stores the data locally and initiates data uploads, whenever a link becomes available.

Component 3 consists a medical server(s) or healthcare server, accessed through the Internet. Moreover, this component could embrace other servers, such as informal caregivers, commercial healthcare providers, and even emergency servers. The medical server commonly composes a service that establishes a communication channel to the patient's application. It assembles the data from the patient application and unites the data into the patient's medical record. The service can allowance admonitions, if reports seem to manifest an anomalous condition.

Numerous researches have been studied in intelligent healthcare system and applications of the nanotechnology. Also, genetic algorithms are already a key player in task assignment. Savić et al. [1] presented a genetic algorithm approach to solve task assignment problem. Lee and Shin [2] developed a task assignment problem in minimizing costs for execution and communication. Mei et al. [3] proposed a task distribution framework to support dynamic reconfiguration of PHMS, by means of task redistribution. This framework consists of a coordinator and a set of facilitators. Task assignment problem in arbitrary processor networks was studied in [4]. Pawar et al. [5] presented a method in optimal task assignment processing based on a genetic algorithm. Bachouch et al. [6] composed an optimization model for task assignment in healthcare.
Hu and Marculescu [7] and Alsalih et al. [8] developed models of the channel directions in processor network. Otto et al. [9] studied about the "Personal Server" in their research work. Paré et al. [10] published a review of home telemonitoring in chronic diseases.

Kang et al. [11] classified stress status based on EEG signals. Sun-Jin [12] designed a smart application that can accurately diagnose and process the current state of the local environment, objects, and persons remotely. Also, Kim et al. [13] developed mobile healthcare application based on the tongue diagnosis.

Kumar et al. [14] proposed a general real-time healthcare monitoring system architecture and summarized the need, challenges, and radio technologies of WBANs, since it is the key factor to achieve low power and low latency. Hanen et al. [15] proposed a mobile medical web service system and implemented a medical cloud multiagent system using Android operating system. Hamdi et al. [16] introduced other system in the management of medical technology. O'Donoghue and Herbert [17] presented the Data Management System (DMS) architecture, which employs an agent-based middleware to intelligently and effectively manage all pervasive medical data sources. Lee et al. [18] composed a management system, which is suitable for diabetes patients by their blood sugar level. Ketan et al. [19] developed a healthcare system for the diabetes patients. Nkenyereye and Jang [20] presented a design in the healthcare web service gateway of the healthcare monitoring system for distance runner.

Sahoo et al. [21] introduced the chief scientific and technical aspects of nanotechnology and discussed some of its potential clinical applications. Omanović-Mikličanin et al. [22] composed the perspective of the nanotechnology applications in medicine. Raffa et al. [23] summarized the progress made in nanotechnology for healthcare. Ali and Abu-Elkheir [24] overviewed a foresight of the healthcare environs and its structural challenges, which are used nanotechnology and nanonetworks. 


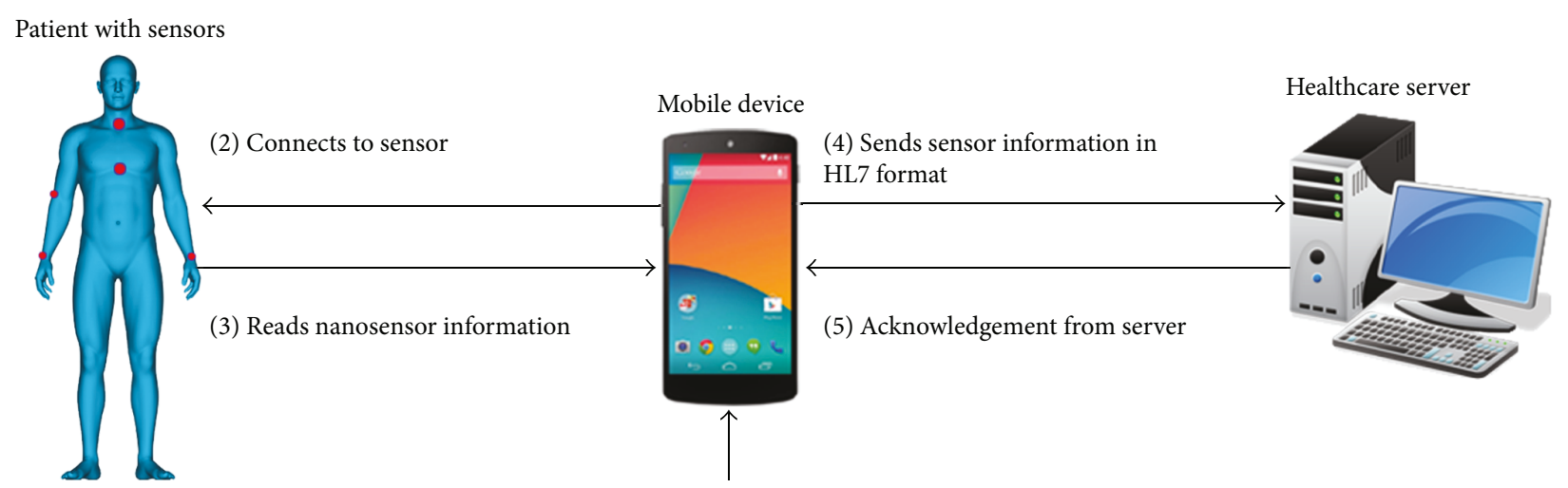

(1) Sensor and Patient information

FIGURE 2: Design of the IHDMS.

Earlier systems were concentrated on obtaining the patient's information irrespective of the resources been used. A raw data produced by these systems were transferred to healthcare server, which caused a lot of overhead of handling this information on the server.

Even though previous researches are composed of various methodologies in the healthcare management system, that is, $[14,17-20]$, the task assignment, that is, [1-6], the healthcare application for the smart phone, that is, $[12,13,15]$, and nanotechnology application for healthcare, that is, [21-24], any work does not perform using these methods together. This study focuses on to developing Intelligent Healthcare Data Management System using nanosensors, which includes task assignment algorithms based on a genetic algorithm, a smart device application, and nanosensors with high sensitivity all together. In addition, genetic algorithm can decrease waiting time in data transpose and demands lower memory while providing the assignment. This paper is arranged as follows. Section 2 explains the objectives and newly proposed methodology. Section 3 presents results and discussion generated from this research work. Section 4 gives conclusions and future works form this study, like the goals achieved and efficiency of the system.

\section{Objectives and Methodology}

The main goal of this study was to design the Intelligent Healthcare Data Management System using nanosensors and develop an application for mobile device. In our proposed system, a device is ubiquitous, and software supplies the real-time patient health information to the caregiver using nanosensors.

An algorithm to obtain the overall methodology used in this paper is as follows:

(i) Design the Intelligent Healthcare Data Management System (IHDMS) using nanosensors.

(ii) Develop a task assignment algorithm (TAA).

(iii) Transform sensor data to HL7.

(iv) Develop an IHDMS application.
2.1. Task Assignment. In the Healthcare Data Management System, a "single task" is a connection between mobile system and a body sensor at a distinct time and assemble the sick person or patient's physiological health information.

If a TAA commends an individual task to the sensor, operation in that begins. In our composed system, the IHDMS connects to target sensor, assembles, and operates the patient's health information, then keep in present information.

In this research, the TAA was developed using genetic algorithm. The genetic algorithm is found on not complicated evolutionary process and consuming pointed option to get optimal results. In genetic algorithm, population of the chromosome was produced.

2.2. Transform of the Nanosensor Data to HL7. After operating the sensor information, it was transformed to the healthcare server by HL7 standard.

An individual HL7 message consists of MSH, PID, and OBX segments. Therefore, these three kinds of segments are produced in our proposed IHDMS.

2.3. An Application for IHDMS. An application programming interface (API) for smart device was composed in the proposed Intelligent Healthcare Data Management System using nanosensors. The IHDMS application based on Android OS and utilized Java program.

The application consists a main page and other subpages, which are unable to get and enter information about sensors, and patient's health information.

\section{Results and Discussion}

3.1. Design of the Intelligent Healthcare Data Management System Using Nanosensors. This section presents the design and performance of the Intelligent Healthcare Data Management System (IHDMS) using nanosensors. In this study, a sick person or patient's health information was assembled from the nanosensors. The assembled health information was operated on the phone and transformed to the HL7 format for transfer to the healthcare server. Figure 2 illustrates the design of the IHDMS for better understanding. 
3.2. Task Assignment Algorithm (TAA). Task assignment algorithm (TAA) is the key component of this study. Primarily, connection between the nanosensor and the mobile device must be set up. There are several predefined nanosensors, which are the user or the caregiver can choose and connect manually to them. User, who is the first time addressed to connect to the nanosensor, should get permission to make a contact in further by an allowed passport. The allowed passport is saved in the system for user's automatic connection afterwards.

Nanosensor information page for connected nanosensors was displayed. In this page, the user can choose scan time and scan daily inputs. Entered information is saved and required in the TAA to produce chromosomes. In our system, input scan time was determined between 10 to 60 seconds, and scan daily input was limited 24 scans daily. This limitation of the scan time and scan daily is reasoned from the current research demands, such as reduce memory usage and save battery of the mobile device.

If there are no nanosensor inputs (scan time and daily scanned inputs) of the system, any chromosome would not be produced by the TAA in the IHDMS. In this case, default values for every inputs are equal to zero (0). Instantly, the application is operated by the user; the TAA is created.

The chromosomes, which are produced by the TAA, consist two variants of chromosomes with distinct bit sizes. These chromosomes are scanned daily and a single hour. In order to produce the chromosomes, the TAA serves two different functions.

The first function operates once in around the clock and produces the daily scan for each sensor. The second function operates every hour and produces the scan per hour chromosome for the whole activated sensors in the current time.

This scan information can be used to produce the scans per hour chromosome for all nanosensors. Twentyfour bit chromosomes will be produced during this process for each sensor. These produced chromosomes create nanosensor's scan information for every hour of the single day. As a single day divided by twenty-four hours, the size of the scans in each day chromosome is equal to twentyfour bit, and the maximum number of the scans in each day for each nanosensor is also twenty-four. So a cell of the chromosome represents a single hour of the day. The cell of the chromosome can get a value 1 or 0 . When the process of the sensor information in the current hour finished, the cell gets a value of 1 bit. When the sensor information will not be processed in the current hour, the cell gets a value of 0 in the chromosome.

Producing these chromosomes is little various from the genetic algorithm-based chromosome generation. In the genetic algorithm, a population of the chromosome was generated, and their fitness was evaluated. Then a novel population through the genetic operations was created. This algorithm is convenient on a powerful system, and there is no limitation on system resources such as memory and battery. The proposed TAA is a genre of the genetic algorithm.

According to the genetic algorithm theory, randomized population of the chromosomes was produced and the chromosomes were rejected based on its fitness. Genetic

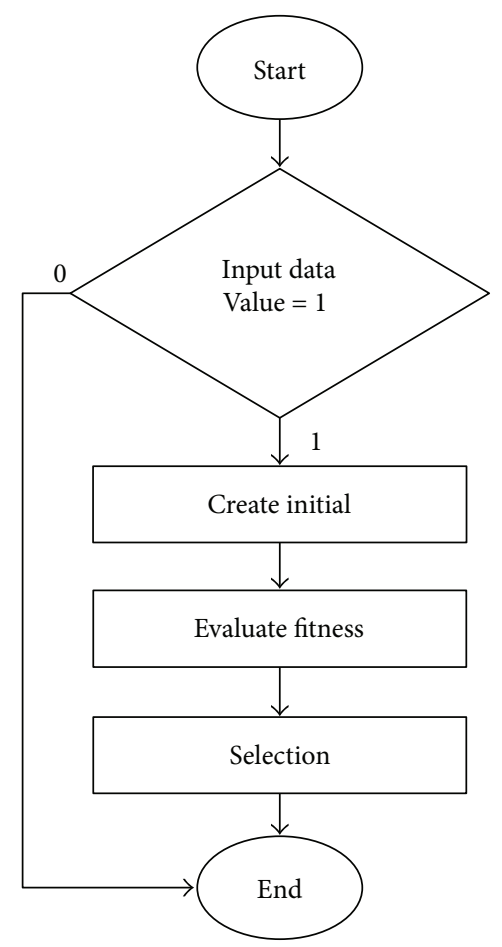

FIgURE 3: Genetic algorithm for task assignment.

$\begin{array}{llllllllllllllllllllllll}1 & 1 & 1 & 1 & 1 & 1 & 1 & 1 & 1 & 1 & 1 & 1 & 0 & 0 & 0 & 0 & 0 & 0 & 0 & 0 & 0 & 0 & 0 & 0\end{array}$

FIGURE 4: Chromosome before randomization.

$\begin{array}{llllllllllllllllllllllll}0 & 0 & 0 & 0 & 1 & 1 & 1 & 0 & 1 & 0 & 1 & 1 & 1 & 1 & 1 & 0 & 1 & 0 & 0 & 1 & 1 & 0 & 0 & 0\end{array}$

FIgURE 5: Chromosome after randomization.

algorithm for the proposed task assignment is presented as a flowchart in Figure 3.

This process consists $n$ number of iterations, randomizations, and adoption. In order to reduce the $n$ number of iterations, randomization, and selection, the TAA was started working from the fitness function of the system.

Figure 4 presents produced chromosomes before randomization.

According to the requirement of the system to obtain randomized health information of the patient, the produced chromosomes were randomized in this study. Figure 5 illustrates produced chromosomes after randomization.

The proposed TAA does not have an assumption of crossover and mutation as in the genetic algorithm. The genetic algorithms generally consist of three fundamental operators: reproduction, crossover, and mutation. The crossover and mutation are utilized to produce a new population of the chromosomes, and a fitness function is used to reject unfit chromosomes. The genetic algorithm is utilized to get the best solution after $n$ number of population generation, fitness selections, crossover, and mutation without specifying 
TABLE 3: MSH segment field information.

\begin{tabular}{lcccc}
\hline Seq. & Len. & Fmt & Opt. & Field name \\
\hline 0 & 3 & & $\mathrm{R}$ & Segment ID = "MSH" \\
1 & 1 & ST & R & Field separator \\
2 & 4 & ST & R & Encoding characters \\
3 & 20 & HD & R & Sending application \\
4 & 20 & HD & R & Sending facility \\
5 & 20 & HD & R & Receiving application \\
6 & 20 & HD & R & Receiving facility \\
7 & 14 & TS & R & Date/time of message \\
8 & 40 & ST & O & Security \\
9 & 7 & CM & R & Message type \\
10 & 20 & ST & R & Message control ID \\
11 & 3 & PT & R & Processing ID \\
12 & 8 & ID & R & Version ID \\
13 & 15 & NM & O & Sequence number \\
14 & 180 & ST & O & Continuation pointer \\
15 & 2 & ID & O & Accept acknowledgment type \\
16 & 2 & ID & O & Application acknowledgment type \\
17 & 2 & ID & O & Country code \\
18 & 6 & ID & O & Character set \\
19 & 60 & CE & O & Principal language of message \\
\hline
\end{tabular}

Practically, all information is entered by the user on primary setup of the application in the IHDMS. A representative result of the MSH message according to Table 3 is shown as below.

MSH||IHDMS|IHDMSMobilePhone|IHDMSMobile Phone|Lab|11101026914414||ORU^R10001075067129 |P|2.4|1|||NE||ASCII|||

The next segment in the HL7 is the PID (Patient Identification) segment. This segment contains 30 different fields (see Table 4), such as patient ID number, sex, address, phone number, marital status, primary language, nationality, citizenship, and religion.

A representative result of the PID segment in a manner corresponding to Table 4 is shown as below.

PID||6804010117||Lee^ Malrey|||||||||||||0803738490 |||||||||||

The next segment after PID is the OBX (Observation) segment. The OBX segment is a part of multiple message types that transmit patient clinical information. 17 different fields of the OBX segment are detailed in Table 5.

A sample result of the OBX segment in a manner corresponding to Table 5 is shown as below.

OBX||ST|ECG Nanosensor||2.675^2.925^2.855^|ECG Nanosensor||||||||||||

Each segment information of the MSH, PID, and OBX are mandatory to produce an accomplished HL7 message that can be delegated to the healthcare server. An individual HL7 message consists of MSH, PID, and OBX segments, which are produced by IHDMS. HL7 messages was transferred to the healthcare server afterward producing process.
TABLE 4: PID segment field information.

\begin{tabular}{|c|c|c|c|c|}
\hline Seq. & Len. & Fmt & Opt. & Field name \\
\hline 0 & 3 & & $\mathrm{R}$ & Segment ID = "PID" \\
\hline 1 & 4 & SI & $\mathrm{O}$ & Set ID_patient ID \\
\hline 2 & 12 & $\mathrm{CX}$ & $\mathrm{O}$ & Patient ID (external ID) \\
\hline 3 & 16 & $\mathrm{CX}$ & $\mathrm{R}$ & Patient ID (internal ID) \\
\hline 4 & 20 & $\mathrm{CX}$ & $\mathrm{O}$ & Alternate patient ID_PID \\
\hline 5 & 48 & $\mathrm{PN}$ & $\mathrm{R}$ & Patient name \\
\hline 6 & 48 & $\mathrm{PN}$ & $\mathrm{O}$ & Mother's maiden name \\
\hline 7 & 14 & TS & $\mathrm{O}$ & Date/time of birth \\
\hline 8 & 1 & IS & $\mathrm{O}$ & Sex \\
\hline 9 & 48 & PN & $\mathrm{O}$ & Patient alias \\
\hline 10 & 1 & IS & $\mathrm{O}$ & Race \\
\hline 11 & 106 & $\mathrm{AD}$ & $\mathrm{O}$ & Patient address \\
\hline 12 & 4 & IS & $\mathrm{O}$ & County code \\
\hline 13 & 20 & $\mathrm{TN}$ & $\mathrm{O}$ & Phone number-home \\
\hline 14 & 20 & $\mathrm{TN}$ & $\mathrm{O}$ & Phone number-business \\
\hline 15 & 20 & $\mathrm{CE}$ & $\mathrm{O}$ & Native language \\
\hline 16 & 1 & IS & $\mathrm{O}$ & Marital status \\
\hline 17 & 3 & IS & $\mathrm{O}$ & Religion \\
\hline 18 & 12 & $\mathrm{CX}$ & $\mathrm{R}$ & Patient account number \\
\hline 19 & 11 & ST & $\mathrm{O}$ & SSN number-patient \\
\hline 20 & 25 & ST & $\mathrm{O}$ & Driver's license number-patient \\
\hline 21 & 9 & $\mathrm{CX}$ & $\mathrm{O}$ & Mother's identifier \\
\hline 22 & 3 & IS & $\mathrm{O}$ & Ethnic group \\
\hline 23 & 20 & ST & $\mathrm{O}$ & Birth place \\
\hline 24 & 2 & ID & $\mathrm{O}$ & Multiple birth indicator \\
\hline 25 & 2 & NM & $\mathrm{O}$ & Birth order \\
\hline 26 & 4 & IS & $\mathrm{O}$ & Citizenship \\
\hline 27 & 60 & $\mathrm{CE}$ & $\mathrm{O}$ & Veterans military status \\
\hline 28 & 80 & $\mathrm{CE}$ & $\mathrm{O}$ & Nationality \\
\hline 29 & 8 & TS & $\mathrm{O}$ & Patient death date and time \\
\hline 30 & 1 & ID & $\mathrm{O}$ & Patient death indicator \\
\hline
\end{tabular}

3.4. The IHDMS Android Application. Once the task assignment algorithm assigns the task to a particular nanosensor, then "Personal Server" is solely responsible for collecting data and events from the wireless body area network (WBAN). The "Personal Server" provides the user interface, controls the WBAN, fuses data and events, and creates unique session archive files. The software is implemented in Java for Android Mobile Operating System. It runs on Mobile Phone with Android OS. The Intelligent Healthcare Data Management System (IHDMS) Application was developed and implemented in this study. Android OS was used in our developed application. Figure 6 illustrated Start Page of the Android application for IHDMS.

A current user's health information can be seen by clicking button "View Information," if it is allowed already. At first time, it will be blank, because there is nobody, and any nanosensor was not allowed. User or caregiver permeates to the system through enter mandatory information by 
TABLE 5: OBX segment field information.

\begin{tabular}{lcccc}
\hline Seq. & Len. & Fmt & Opt. & Field name \\
\hline 0 & 3 & & R & Segment ID = "OBX" \\
1 & 10 & SI & O & Set ID-OBX \\
2 & 2 & ID & R & Value type \\
3 & 20 & CE & R & Observation identifier \\
4 & 20 & ST & O & Observation Sub-ID \\
5 & 10 & NM & R & Observation value \\
6 & 20 & CE & R & Units \\
7 & 10 & ST & O & References range \\
8 & 5 & ID & O & Abnormal flags \\
9 & 5 & NM & O & Probability \\
10 & 2 & ID & O & Nature of abnormal test \\
11 & 1 & ID & O & Result status \\
12 & 14 & TS & O & Date of last normal values \\
13 & 20 & ST & O & User defined access checks \\
14 & 14 & TS & O & Date/time of the observation \\
15 & 60 & CE & O & Producer's ID \\
16 & 80 & CN & O & Responsible observer \\
17 & 60 & CE & O & Observation method \\
\hline
\end{tabular}

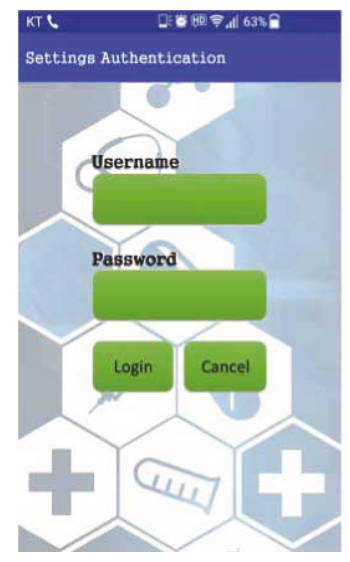

FIgUre 7: Settings Authentication page.

"Options" button. Figure 7 shows a Settings Authentication page by clicking "Options" button in Figure 8 .

After updating the patient information, the current page Patient Information is redirected to the previous page of Patient Settings, as illustrated in Figure 9. Next step is to set the nanosensor information. A Page of the Set Selected Nanosensors in Android Application is illustrated in Figure 10. Figure 11 presents Nanosensor Settings Page and result of the ECG nanosensor.

Acquired nanosensor data is sent to micro gateway, and the micro gateway data is sent to mobile device. Mobile device application displays received information to the user. Figure 12 illustrated data receiving structure and result of the Blood pressure, oxygen saturation in the mobile device.

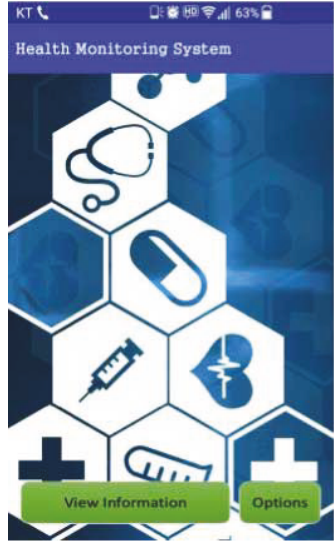

FIgure 8: Start page of the Android application in Health Monitoring System.

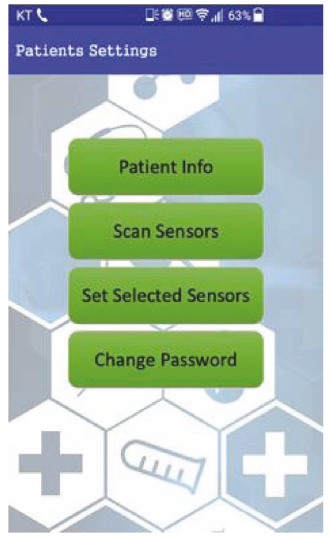

(a) Patient Settings page

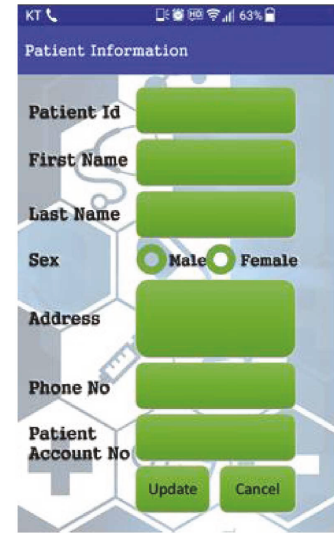

(b) Patient Information page
Figure 9: Patient Settings and Patient Information pages.

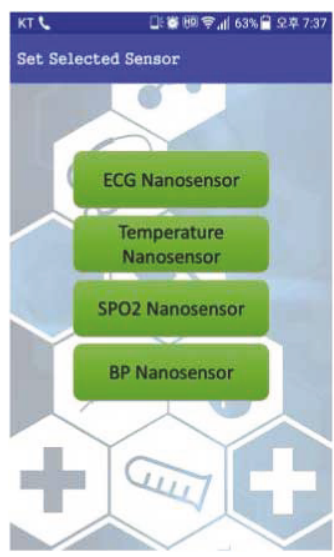

Figure 10: Pages of the set nanosensors and nanosensor settings.

\section{Conclusions and Future Works}

The proposed Intelligent Healthcare Data Management System dynamically assembles the nanosensor information of the sick person or patient and operates it on the smart phone. 


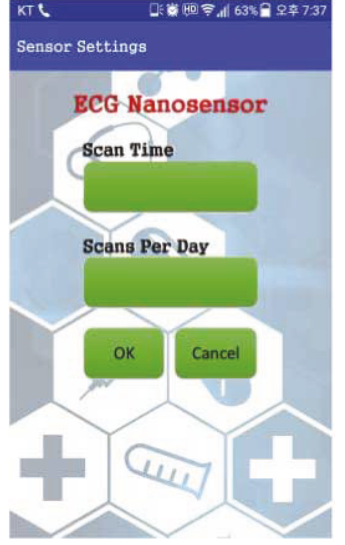

(a) Nanosensor settings page of the EGG nanosensor

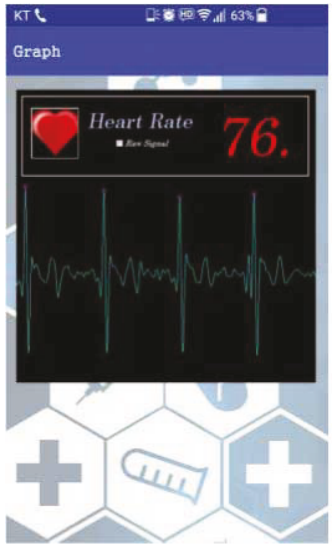

(b) Result page of the ECG nanosensor
Figure 11: Nanosensor settings and result page of the ECG nanosensor.

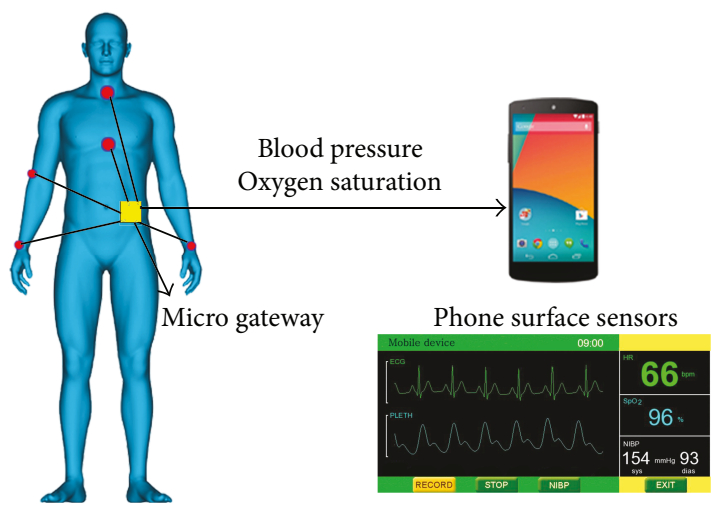

FIGURE 12: Data receiving structure and result of the Blood pressure, oxygen saturation in the mobile device.

It conducts the patient's health information in a superior prolific process.

This system also transforms raw data of the user from nanosensors to HL7 format and transfers the encrypted HL7 data to a remote healthcare server.

The main challenge faced by this system is in terms of the limited resources on the mobile environment. Similar to other applications operating in a mobile environment, the IHDMS could be deeply affected by context changes and scarcity of mobile platform's resource like network bandwidth, battery power, and computational power of handhelds.

This converted data dispatches to a server of its system. The battery lifetime of the facility is feasible to increase, the memory usage is less than $100 \mathrm{~KB}$, and it operates all data by employing few and far between resources. Moreover, the proposed system decreases the waiting time in the transposing data, and secured channel was used for the server of the healthcare center in the running HL7 format data.

In the future, we will carry on this research in order to consummate the main point interfacing facilitator, to relate with other varying types of patient care devices that appear in medical industry. Also, the task assignment algorithm (TAA) can be amplified to utilize the motion nanosensor of the mobile device and apply this information in assigning task. We desire that the HL7 middleware library package will be maturing, to become embedded software that can be used in ubiquitous healthcare devices.

\section{Conflicts of Interest}

The authors declare that they have no conflicts of interest.

\section{Acknowledgments}

This research is funded by research funds of Wonkwang University in 2016. This research was supported by Next-Generation Information Computing Development Program through the National Research Foundation of Korea (NRF) funded by the Ministry of Science, ICT \& Future Planning (2014M3C4A7030503). Also, this study was supported by the National Research Foundation of Korea (NRF) Grant funded by the Korea government (MSP) no. 2017R1A2B400667.

\section{References}

[1] A. Savić, D. Tošić, M. Marić, and J. Kratica, "Genetic algorithm approach for solving the task assignment problem," Serdica Journal of Computing, vol. 2, pp. 267-276, 2008.

[2] C.-H. Lee and K. G. Shin, "Optimal task assignment in homogeneous networks," IEEE Transactions on Parallel and Distributed Systems, vol. 8, no. 2, pp. 119-129, 1997.

[3] H. Mei, B. J. Van Beijnum, P. Pawar, I. Widya, and H. Hermens, "A*-based task assignment algorithm for context-aware mobile patient monitoring systems," in 2009 15th IEEE International Conference on Embedded and Real-Time Computing Systems and Applications, Beijing, China, 2009.

[4] B. Zhao, M. Wang, Z. Shao, J. Cao, K. C. C. Chan, and J. Su, "Topology aware task allocation and scheduling for real-time data fusion applications in networked embedded sensor systems," in 2008 14th IEEE International Conference on Embedded and Real-Time Computing Systems and Applications, pp. 293-302, Kaohsiung, Taiwan, 2008.

[5] P. Pawar, H. Mei, I. Widya, B.-J. van Beijnum, and A. van Halteren, "Context-aware task assignment in ubiquitous computing environment - a genetic algorithm based approach," in 2007 IEEE Congress on Evolutionary Computation, pp. 26952702, Singapore, Singapore, 2007.

[6] R. B. Bachouch, A. Guinet, and S. Hajri-Gabouj, "An optimization model for task assignment in home health care," in 2010 IEEE Workshop on Health Care Management (WHCM), Venice, Italy, 2010.

[7] J. Hu and R. Marculescu, "Energy- and performance-aware mapping for regular NoC architectures," IEEE Transactions on Computer-Aided Design of Integrated Circuits and Systems, vol. 24, no. 4, pp. 551-562, 2005.

[8] W. Alsalih, S. Akl, and H. Hassanein, "Energy-aware task scheduling: towards enabling mobile computing over MANETs," in 19th IEEE International Parallel and Distributed Processing Symposium, Denver, CO, USA, 2005.

[9] C. Otto, A. Milenkovic, C. Sanders, and E. Jovanov, "System architecture of a wireless body area sensor network for ubiquitous health monitoring," Journal of Mobile Multimedia, vol. 1, pp. 307-326, 2006. 
[10] G. Paré, M. Jaana, and C. Sicotte, "Systematic review of home telemonitoring for chronic diseases: the evidence base," Journal of the American Medical Informatics Association, vol. 14, no. 3, pp. 269-277, 2007.

[11] J.-S. Kang, G. Jang, and M. Lee, "Stress status classification based on EEG signals," The Journal of The Institute of Internet, Broadcasting and Communication, vol. 16, no. 3, pp. 103-108, 2016.

[12] O. Sun-Jin, "Design of a smart application for remote diagnosis in ubiquitous computing environment," The Journal of The Institute of Internet, Broadcasting and Communication, vol. 16, no. 4, pp. 81-87, 2016.

[13] J. Kim, J. So, W. Choi, and K.-H. Kim, "Development of a mobile healthcare application based on tongue diagnosis," The Journal of The Institute of Internet, Broadcasting and Communication, vol. 16, no. 4, pp. 65-72, 2016.

[14] V. Kumar, B. Gupta, and S. K. Ramakuri, "Wireless body area networks towards empowering real-time healthcare monitoring: a survey," International Journal of Sensor Networks, vol. 22 , no. 3, pp. 177-187, 2016.

[15] J. Hanen, Z. Kechaou, and M. B. Ayed, "An enhanced healthcare system in mobile cloud computing environment," Vietnam Journal of Computer Science, vol. 3, no. 4, pp. 267277, 2016.

[16] N. Hamdi, R. Oweis, H. A. Zraiq, and D. A. Sammour, “An intelligent healthcare management system: a new approach in work-order prioritization for medical equipment maintenance requests," Journal of Medical Systems, vol. 36, no. 2, pp. 557-567, 2012.

[17] J. O’Donoghue and J. Herbert, "Data management within mHealth environments: patient sensors, mobile devices, and databases," Journal of Data and Information Quality, vol. 4, no. 1, article 5, pp. 1-20, 2012.

[18] M. Lee, T. M. Gatton, and K.-K. Lee, "A monitoring and advisory system for diabetes patient management using a rulebased method and KNN," Sensors, vol. 10, no. 4, pp. 39343953, 2010.

[19] D. Ketan, R. R. Bodhe, A. N. Sawant, and A. Kazi, "Proposed mobile based healthcare system for patient diagnosis using android OS," International Journal of Computer Science and Mobile Computing, vol. 3, no. 5, pp. 422-427, 2014.

[20] N. Lionel and J. Jong-Wook, “Addressing concurrency design for HealthCare Web Service Gateway in remote healthcare monitoring system," International Journal of Advanced Smart Convergence, vol. 5, no. 3, pp. 32-39, 2016.

[21] S. K. Sahoo, S. Parveen, and J. J. Panda, "The present and future of nanotechnology in human health care," Nanomedicine: Nanotechnology, biology, and Medicine, vol. 3, no. 1, pp. 20-31, 2007.

[22] E. Omanović-Mikličanin, M. Maksimović, and V. Vujović, "The future of healthcare: nanomedicine and internet of nano things," Folia Medica Facultatis Medicinae Universitatis Saraeviensis, vol. 50, no. 1, pp. 23-28, 2015.

[23] V. Raffa, O. Vittorio, C. Riggio, and A. Cuschieri, "Progress in nanotechnology for healthcare," Minimally Invasive Therapy \& Allied Technologies, vol. 19, no. 3, pp. 127-135, 2010.

[24] N. A. Ali and M. Abu-Elkheir, "Internet of nano-things healthcare applications: requirements, opportunities, and challenges," in 2015 IEEE 11th International Conference on Wireless and Mobile Computing, Networking and Communications (WiMob), Abu Dhabi, United Arab Emirates, 2015. 


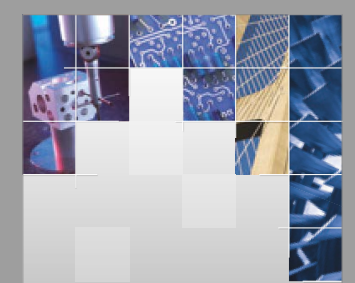

\section{Enfincering}
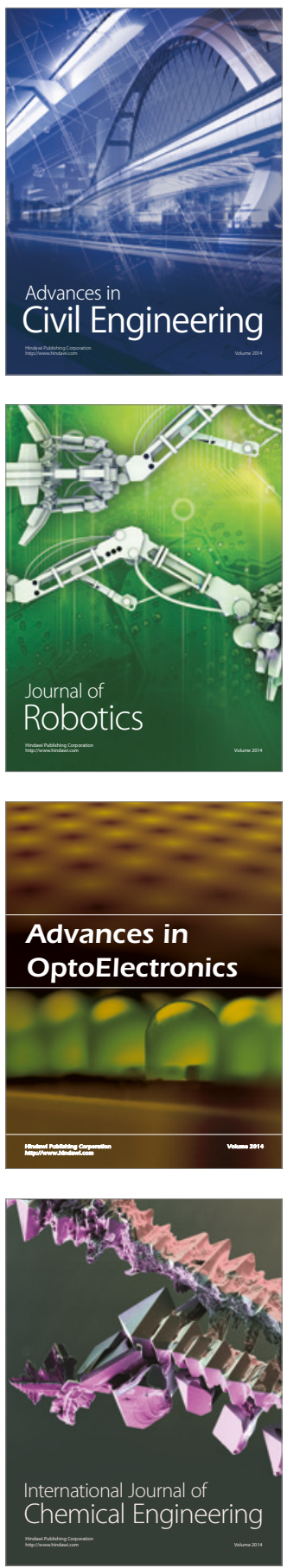

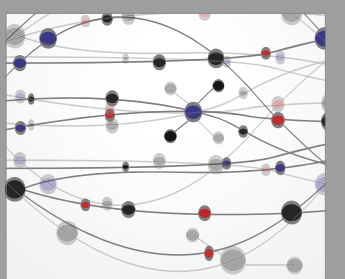

The Scientific World Journal

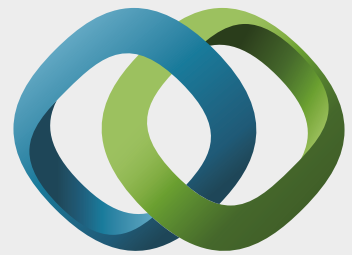

\section{Hindawi}

Submit your manuscripts at

https://www.hindawi.com
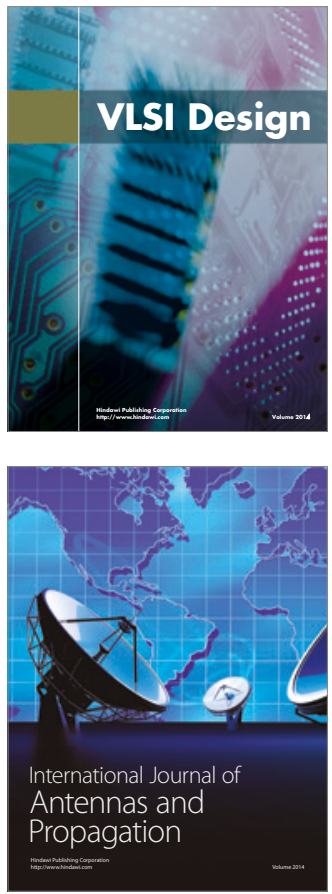

\section{Rotating}

Machinery
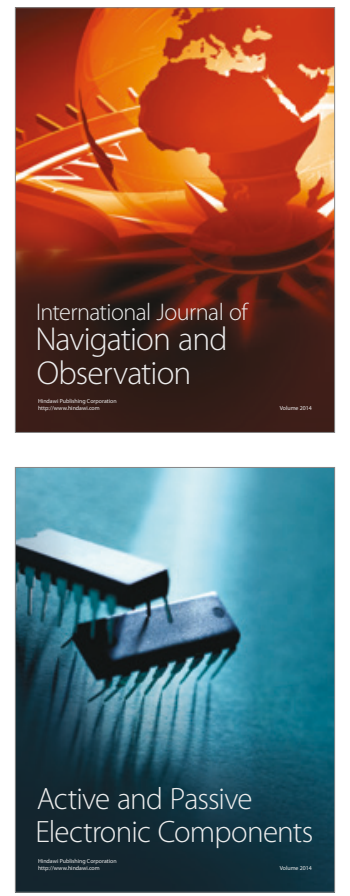
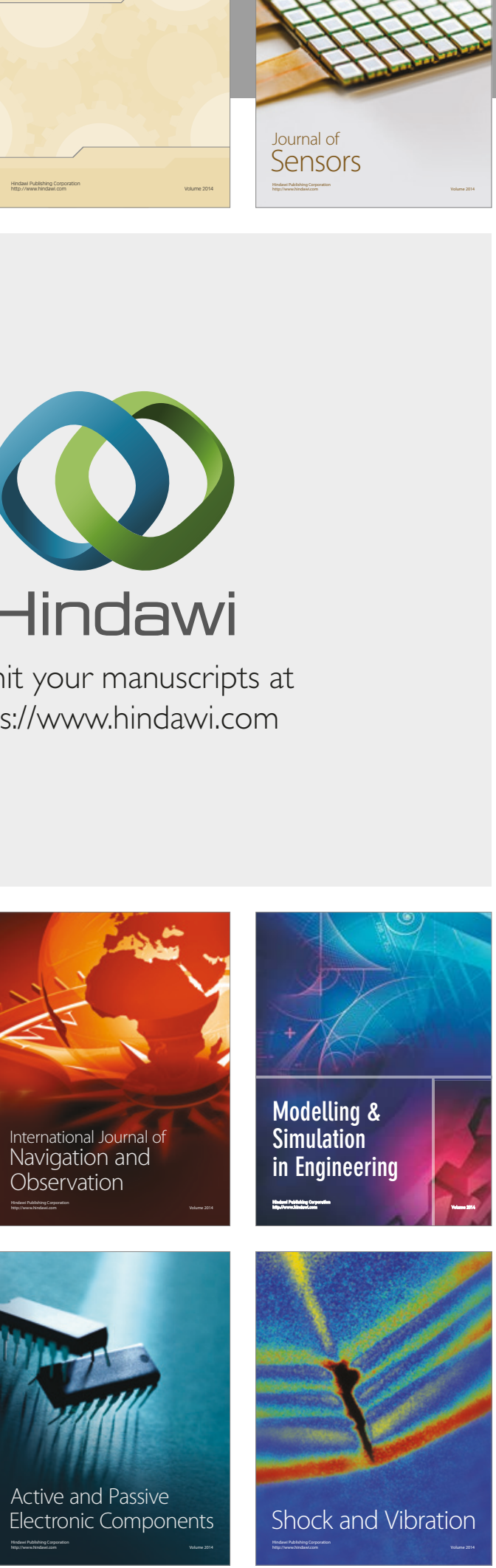
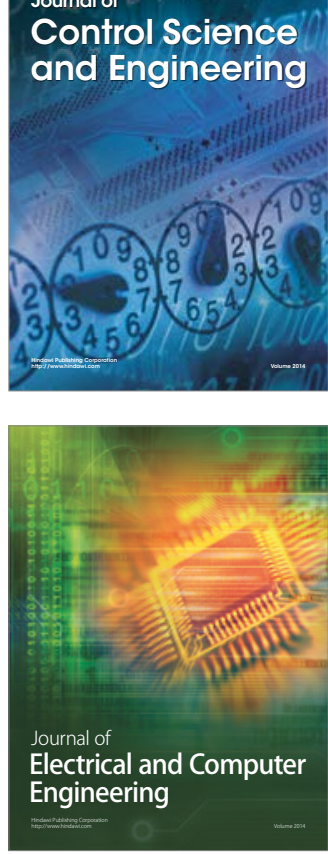

Distributed

Journal of

Control Science

and Engineering
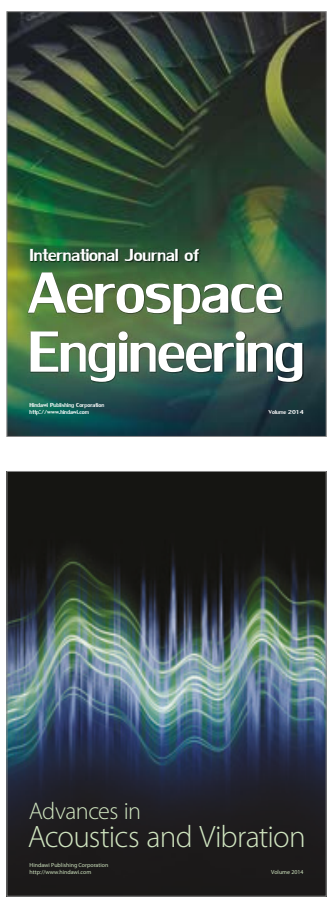

Sensor Networks 